The Astrophysical Journal, 678:376-384, 2008 May 1

(C) 2008. The American Astronomical Society. All rights reserved. Printed in U.S.A.

\title{
THE INTERMEDIATE POLAR EI UMa: A PREPOLAR CATACLYSMIC VARIABLE
}

\author{
TAMARA W. REIMER \\ Department of Astronomy, San Diego State University, 5500 Campanile Drive, San Diego, CA 92182-1221; tamara.reimer@gmail.com \\ William F. WeLSH \\ Department of Astronomy, San Diego State University, 5500 Campanile Drive, San Diego, CA 92182-1221; wfw@sciences.sdsu.edu \\ KoJi Mukai \\ Astrophysics Science Division, University of Maryland, Baltimore County; also NASA Goddard Space Flight Center, Code 662, \\ Greenbelt, MD 20771; mukai@milkyway.gsfc.nasa.gov \\ AND \\ F. A. Ringwald \\ Department of Physics, California State University, Fresno, 2345 East San Ramon Avenue, M/S MH37, Fresno, \\ CA 93740-8031; ringwald@csufresno.edu \\ Received 2007 June 8; accepted 2008 February 3
}

\begin{abstract}
We present optical and X-ray time series photometry of EI UMa that reveal modulation at 746 and $770 \mathrm{~s}$, which we interpret as the white dwarf spin and spin-orbit sidebands. These detections, combined with previous X-ray studies, establish EI UMa as an intermediate polar. We estimate the mass accretion rate to be $\sim 3.6 \times 10^{17} \mathrm{~g} \mathrm{~s}^{-1}$, which is close to, and likely greater than, the critical rate above which dwarf nova instabilities are suppressed. We also estimate the white dwarf to have a large magnetic moment $\mu>(3.4 \pm 0.2) \times 10^{33} \mathrm{G} \mathrm{cm}^{3}$. The high mass accretion rate and magnetic moment imply the existence of an accretion ring rather than a disk, and along with the relatively long orbital period, these suggest that EI UMa is a rare example of a prepolar cataclysmic variable.
\end{abstract}

Subject headings: accretion, accretion disks — novae, cataclysmic variables - stars: dwarf novae stars: individual (EI UMa)

\section{INTRODUCTION}

Cataclysmic variables are binary star systems in which gas is gravitationally stripped from a companion star and accreted onto a white dwarf, typically via an accretion disk. In intermediate polar systems (also known as "DQ Her stars") the accretion disk is disrupted by the magnetic field of the white dwarf. Transferred material forms a disk at large radii, but follows the magnetic field of the white dwarf when inside the white dwarf's magnetosphere. The rotation of the white dwarf and the magnetically channeled accretion flow creates periodic modulations in the observed flux. Patterson (1994) lists six observational criteria used to identify an intermediate polar: (1) a stable optical period $P_{\text {spin }}$ that is less than the orbital period; (2) a stable X-ray period at or near the optical period; (3) pulsations in the He II emission lines; (4) circular polarization; (5) a sideband period in the optical and/or X-ray, usually on the long-period side of the main signal; and (6) a very hard X-ray spectrum with low-energy absorption. A cataclysmic variable need not exhibit all six of these characteristics to be an intermediate polar (henceforth abbreviated as IP).

EI Ursa Majoris, also known as PG 0834+488, was discovered in the Palomar-Green Survey of objects with ultraviolet excesses, and subsequently found to be a cataclysmic variable (Green et al. 1982). $\mathrm{H} \alpha$ radial velocities show the system to have an orbital period of $6.434 \mathrm{hr}$ (Thorstensen 1986). Early observations suggested this object exhibits some of the characteristics of an IP. Green et al. observed that the system has an unusually strong He II $\lambda 4686$ line. Cook (1985) noted that the system exhibits a strong hard X-ray flux. The early X-ray observations failed to show any stable periodic modulation, however, leading Cook to suggest that EI UMa was instead a dwarf nova. This classification currently persists (e.g., Ritter \& Kolb 2003), despite the lack of observed outbursts for this system (see Fig. 1). Recent X-ray observations support classification of EI UMa as an IP, and not as a dwarf nova. Pandel et al. (2005) argue for the IP status based on XMM-Newton X-ray spectra. They also found EI UMa to have a low UV luminosity, and suggest this is a result of the missing inner accretion disk. Baskill et al. (2005) claim EI UMa is an IP based on its $A S C A$ $\mathrm{X}$-ray spectral characteristics, and most importantly, the discovery of a $741.6 \pm 5.4$ s periodicity with an amplitude of $(8 \pm 1) \%$.

EI UMa has thus satisfied Patterson's conditions 2 and 6 for being an IP. If a stable optical period were found, the case for EI UMa's IP status would be much strengthened. We therefore collected $84 \mathrm{hr}$ of optical photometry (see $\S 2$ ) to search for oscillations near the X-ray period discovered by Baskill et al. We present the detection of such optical oscillations in $\S 3$ of this paper, and present a second detection of the X-ray period using archival $X M M$-Newton data in $\S 4$. In $\S 5$ we discuss our search for longer timescale phenomena, including ellipsoidal modulations and dwarf nova outbursts. We examine EI UMa as an IP in $\S 6$, estimating its distance, mass accretion rate, and magnetic field strength, and we summarize our results in $\S 7$.

\section{OPTICAL OBSERVATIONS}

We observed EI UMa on 16 nights between 2003 February and 2005 December using the $1 \mathrm{~m}$ telescope and CCD at San Diego State University's Mount Laguna Observatory. An observation $\log$ is presented in Table 1. Observations on the first nine nights looked for modulations on the spin period and harmonics; this set included five nights of $V$-band data, two nights of $U$-band data, and two nights of $B$-band data. Observations during the last seven nights looked for variability on the longer orbital timescale and used an $R$-band filter. Images were bias-subtracted and 


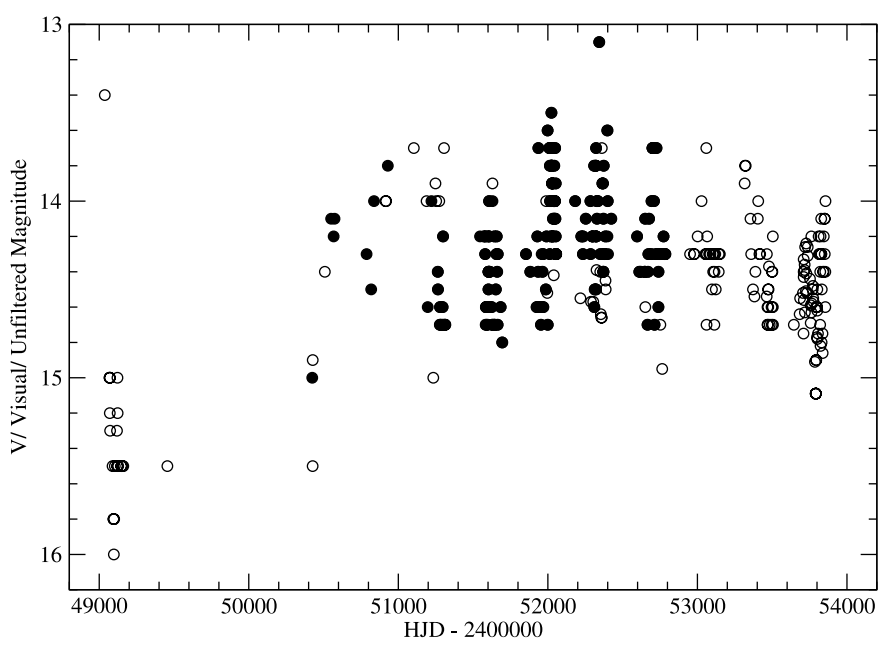

FIG. 1.- Light curve of EI UMa from 1993 to 2006, as provided by the AAVSO (open circles) and the VSNet (solid circles; see T. Kato \& D. Nogami 2003 at VSNet emailing list, http://www.kusastro.kyoto-u.ac.jp/vsnet). Note the lack of dwarf nova outbursts.

flat-field-corrected using standard IRAF data reduction routines, and light curves were extracted by performing aperture photometry with the APPHOT package. A large aperture of $4.8^{\prime \prime}$ was chosen for the $R$-band observations to minimize the night-to-night changes in comparison star light curves. The calibration of the $R$-band light curves is accurate to $\sim 2 \%$, based on the relative flux of the check star. Misselt (1996) gives $B V R$ magnitudes for EI UMa ( $B=14.90, V=14.78$, and $R=14.55$ ) and nearby field stars. Differential light curves from the first four nights use Misselt's star 1 (M1; also star 21 in Henden \& Honeycutt 1997). The remaining 12 light curves use Misselt's star 3 (M3; star 10 in Henden \& Honeycutt), and Misselt's stars 1 and 4 were used as check stars. Nightly mean $V$-, $B$-, and $R$-band magnitudes for EI UMa are presented in Table 2. Comparison magnitudes in the $U$-band were not available. Figure 2 shows our $U, B$, and $V$ differential flux light curves. The most obvious feature is the incessant flickering, a defining characteristic of accretion-powered objects. The rms of the red noise flickering in the differential fluxes is typically $\sim 5 \%$. This rms has not been corrected for observational noise, and thus is slightly overestimated.

\section{THE OPTICAL PERIODICITIES}

\subsection{Time Series Analysis}

Power spectra were computed to search for periodicities near the previously detected X-ray periodicity $(741.6 \pm 5.4 \mathrm{~s}$; Baskill et al. 2005). Before Fourier transformation, the light curves were detrended to minimize low-frequency red noise leakage. We used a sliding boxcar to remove power on timescales longer than 45 minutes. Other detrending methods were tried as a check for robust periodicity; this included subtracting off only the mean or a linear fit to the light curve. Power spectra for 2003 February 1, 2 , and 21 each show a significant signal near $745 \mathrm{~s}$. The LombScargle periodogram (Press et al. 2003) yields a false alarm probability of $4 \times 10^{-18}$ for the February 21 signal. Although this formal probability is meaningless in the presence of red noise, much of the red noise is removed by the prewhitening (detrending) of the light curve, and the extremely small false alarm probability shows that the detection is highly significant. Six additional nights exhibit weaker, but measurable, signals; these signals are not all at the same period, but range between 747 and $815 \mathrm{~s}$. The average amplitude of the periodicity is $\sim 1 \%$. The remaining seven light curves show no detectable periodicities in the period range between 700 and $850 \mathrm{~s}$. No significant shorter period signal was detected in any light curve, indicating no strong harmonics are present. For nights when a periodicity was not detected, amplitude limits were obtained by injecting "tracer" sinusoids of different frequencies and phases into the light curves. The detection limit threshold was defined as the amplitude at which two out of five injected sinusoids were no longer apparent above the noise in the power spectrum. Figure 3 shows the stronger detections, Figure 4 shows examples of nondetections and tentative detections, and the periods and amplitudes are given in Table 2.

\subsection{Spin and Sideband Periods}

The observed optical periods fall into three groups: 747, 760770 , and $813 \mathrm{~s}$. The $747 \mathrm{~s}$ periodicity is seen only in the two $U$-band observations (separated by 2 years). This agrees with the $741.6 \pm 5.4 \mathrm{~s}$ X-ray periodicity seen by Baskill et al. (2005), and we associate these with the spin period of the white dwarf. Combining the $U$-band and X-ray measurements, we derive a weighted average spin period of $745.7 \pm 2.7 \mathrm{~s}$. We note that the two $U$-band

TABLE 1

ObSeRvation Log

\begin{tabular}{|c|c|c|c|c|c|c|}
\hline Night & Filter & UT Start Time & $\begin{array}{l}\text { Duration } \\
\text { (hr: } \mathrm{mm})\end{array}$ & $\begin{array}{r}\text { Aperture } \\
(\operatorname{arcsec})\end{array}$ & $\begin{array}{l}\text { Seeing } \\
(\operatorname{arcsec})\end{array}$ & Comparison Star \\
\hline 2003 Feb $1 \ldots \ldots \ldots \ldots \ldots \ldots$ & V & $09: 26$ & $3: 33$ & 1.6 & 1.0 & M1 \\
\hline 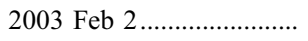 & $V$ & $10: 21$ & $2: 41$ & 2.0 & 3.1 & M1 \\
\hline 2003 Feb $19 \ldots \ldots \ldots \ldots \ldots . .$. & $B$ & $05: 48$ & $6: 19$ & 1.6 & 1.2 & M1 \\
\hline 2003 Feb $21 \ldots \ldots \ldots \ldots$ & V & $03: 50$ & $7: 53$ & 2.0 & 2.6 & M1 \\
\hline 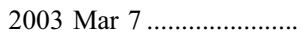 & $U$ & $03: 38$ & $7: 52$ & 2.0 & 1.3 & M3 \\
\hline 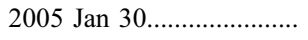 & $V$ & $08: 47$ & $4: 23$ & 1.6 & 1.2 & M3 \\
\hline 2005 Mar $12 \ldots \ldots \ldots \ldots \ldots \ldots$ & $V$ & $04: 22$ & $7: 51$ & 1.6 & 1.4 & M3 \\
\hline 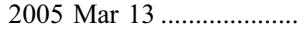 & $U$ & $03: 42$ & $8: 09$ & 2.4 & 1.9 & M3 \\
\hline 2005 Jun 7 & $B$ & $04: 50$ & $1: 18$ & 2.0 & 2.4 & M3 \\
\hline 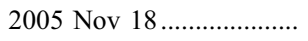 & $R$ & $08: 55$ & $4: 14$ & 4.8 & 2.2 & M3 \\
\hline 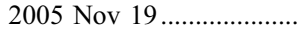 & $R$ & $07: 52$ & $5: 39$ & 4.8 & 3.1 & M3 \\
\hline 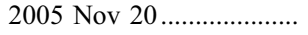 & $R$ & $07: 44$ & $5: 43$ & 4.8 & 2.6 & M3 \\
\hline 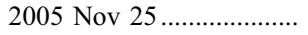 & $R$ & $07: 29$ & $6: 25$ & 4.8 & 3.0 & M3 \\
\hline 2005 Dec $2 \ldots \ldots \ldots \ldots \ldots \ldots \ldots$ & $R$ & $07: 54$ & $5: 39$ & 4.8 & 2.7 & M3 \\
\hline 2005 Dec 4 & $R$ & $07: 10$ & $6: 22$ & 4.8 & 4.8 & M3 \\
\hline 2005 Dec 5 & $R$ & $07: 10$ & $3: 58$ & 4.8 & 3.5 & M3 \\
\hline
\end{tabular}


TABLE 2

Observed Properties of EI UMa

\begin{tabular}{|c|c|c|c|c|c|}
\hline Night & Filter & Mean Magnitude & $\begin{array}{c}\text { rms Flickering } \\
(\%)\end{array}$ & $\begin{array}{l}\text { Period } \\
\quad(\mathrm{s})\end{array}$ & $\begin{array}{c}\text { Amplitude } \\
\text { (\%) }\end{array}$ \\
\hline 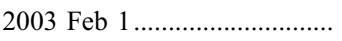 & $V$ & 14.58 & 4.4 & $770 \pm 3$ & $1.3 \pm 0.2$ \\
\hline 2003 Feb 2 & $V$ & 14.70 & 4.3 & $813 \pm 7$ & $1.2 \pm 0.2$ \\
\hline 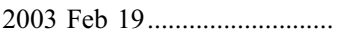 & $B$ & 14.64 & 6.4 & $\ldots$ & $<0.7$ \\
\hline 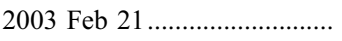 & V & 14.48 & 3.8 & $768 \pm 2$ & $0.9 \pm 0.1$ \\
\hline 2003 Mar 7 ................................. & $U$ & $\ldots$ & 6.0 & $748 \pm 5$ & $0.7 \pm 0.3$ \\
\hline 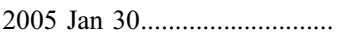 & V & 14.47 & 2.9 & $\ldots$ & $<0.6$ \\
\hline 2005 Mar 12 & $V$ & 14.48 & 3.2 & $765 \pm 3$ & $0.5 \pm 0.1$ \\
\hline 2005 Mar 13 ............................. & $U$ & $\ldots$ & 4.8 & $747 \pm 4$ & $1.0 \pm 0.3$ \\
\hline 2005 Jun 7 & $B$ & 15.00 & 3.5 & $\ldots$ & $<1.0$ \\
\hline 2005 Nov $18 \ldots \ldots \ldots \ldots \ldots \ldots \ldots$ & $R$ & 14.18 & 3.1 & $\ldots$ & $<0.6$ \\
\hline 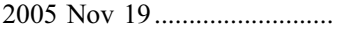 & $R$ & 14.16 & 2.9 & $\ldots$ & $<0.5$ \\
\hline 2005 Nov $20 \ldots \ldots \ldots \ldots \ldots \ldots \ldots$ & $R$ & 13.87 & 6.1 & $812 \pm 7$ & $0.4 \pm 0.1$ \\
\hline 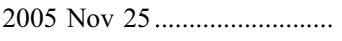 & $R$ & 13.99 & 4.1 & $\ldots$ & $<0.5$ \\
\hline 2005 Dec 2 .................................. & $R$ & 14.24 & 4.1 & $\ldots$ & $<0.8$ \\
\hline 2005 Dec 4 & $R$ & 14.44 & 7.2 & $815 \pm 5$ & $1.0 \pm 0.4$ \\
\hline 2005 Dec 5 & $R$ & 14.46 & 5.4 & $760 \pm 6$ & $1.1 \pm 0.3$ \\
\hline
\end{tabular}

signal detections are not convincing by themselves, but are statistically significant when viewed in combination with the X-ray and the other optical signals.

We identify the stronger 760-770 s oscillation as the first-order sideband period $P_{\text {side }}$ given by $P_{\text {side }}^{-1}=P_{\text {spin }}^{-1}-P_{\text {orb }}^{-1}$. Using the orbital period of $6.434 \pm 0.007 \mathrm{hr}$ (Thorstensen 1986), the sideband period for this system should be $770 \pm 3 \mathrm{~s}$. Our light curves have just enough resolution to distinguish the spin and sideband periodicity, as sinusoid injection simulations give a resolution of
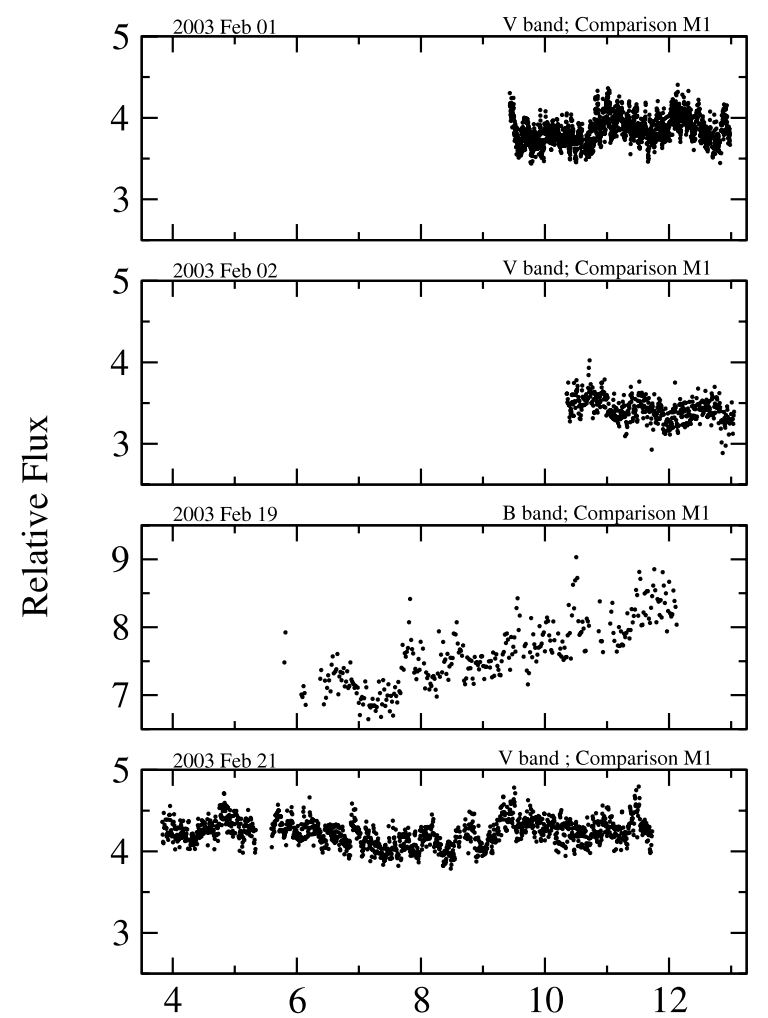

$\sim 20$ s. The discovery of the optical spin and sideband periodicities (criteria [1] and [5] in Patterson 1994) combined with the X-ray periodicities found by Baskill et al. (2005) and also in this work (see $\S 4$ ) provide compelling evidence that EI UMa is an intermediate polar.

Intermediate polars are known to exhibit other sideband periods, so we searched for - but failed to confirm - periodicities at other sidebands. The third-order sideband $P_{\text {side }}^{-1}=P_{\text {spin }}^{-1}-3 P_{\text {orb }}^{-1}$ at $821 \pm 3 \mathrm{~s}$ is in the vicinity of the $813 \mathrm{~s}$ period seen on two, and

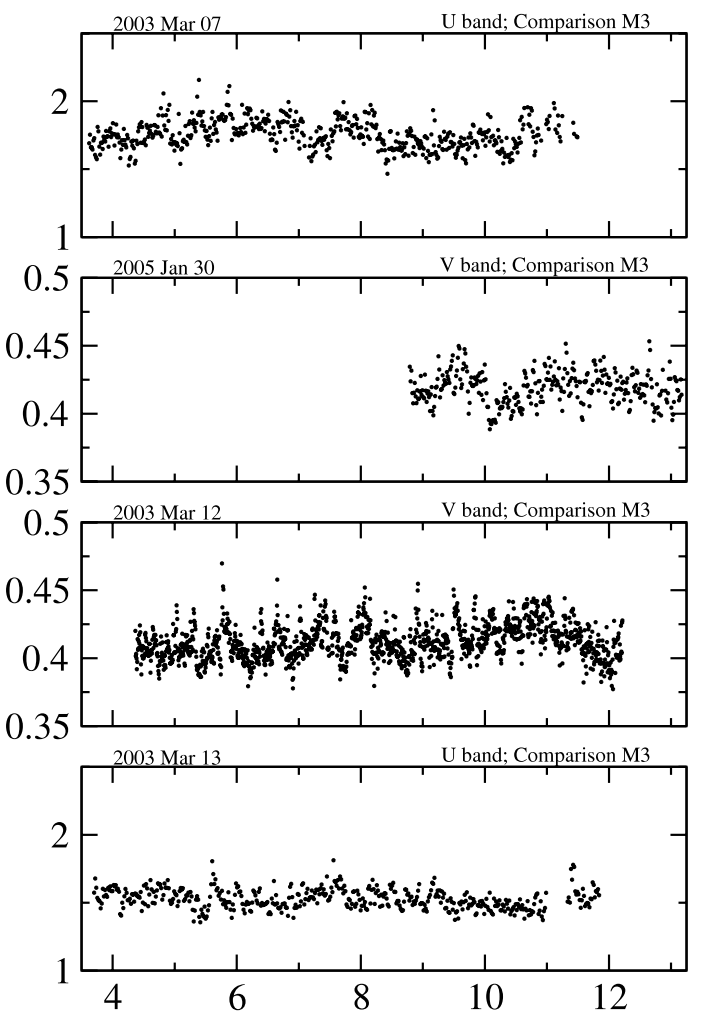

Time (UT)

FIG. 2.- $U-, B$-, and $V$-band light curves of EI UMa. The time is in hours, and the flux is relative to comparison stars from Misselt (1996). The most notable characteristic is the random flickering, a few percent in amplitude. 


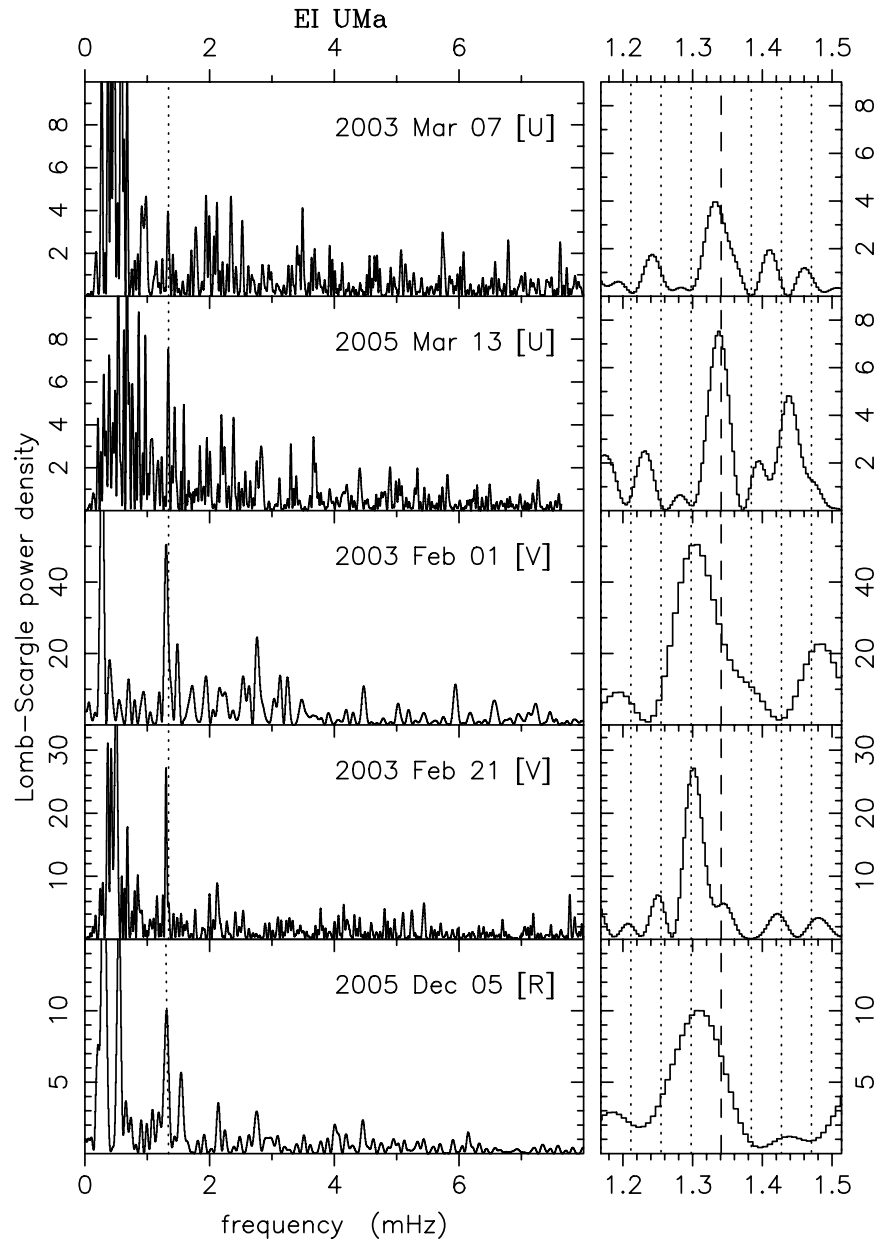

FIG. 3.- Power spectra of prewhitened optical light curves, 10 times oversampled, showing spin and first sideband periods signals. $U$-band power spectra in the top two panels show signals at the $745 \mathrm{~s}$ spin period, while the $V$ - and $R$-band power spectra in the bottom panels show signals consistent with the first sideband, at $770 \mathrm{~s}$. The left-hand panels show the low-frequency part of the power spectra, and the right-hand panels zoom in on the region between 680 and $825 \mathrm{~s}$. A dashed line marks the spin period and dotted lines mark the upper and lower spinorbit sidebands.

possibly three, occasions. However, the agreement is not good enough to firmly associate these with the third sideband. The bottom three panels in Figure 4 show the possible third-order sidebands detections.

The presence of an orbital sideband requires a reprocessing site that is locked in the rotating binary frame, such as the bright spot (Hassall et al. 1981) or the secondary star (Patterson \& Price 1981). Interestingly, the spin period is seen only in our $U$-band light curves and the sidebands are seen only in the $V$ and $R$ bands, where reprocessed radiation is more likely to be seen. Systems that similarly exhibit strong sideband periods with spin periods that are hard to detect at optical wavelengths include V1223 Sgr (Osborne et al. 1985) and YY Dra (Patterson et al. 1992). In the latter system, the $U$ band is the only optical wavelength in which the quiescent spin period is even marginally visible (Haswell et al. 1997).

\subsection{Signal Amplitude and Stability}

Since the light curves of cataclysmic variables are subject to flickering and apparent short-lived periodicities, it is important to establish the stability of any potential spin/sideband period signal (e.g., see Warner 1989). Periodicities were detected in nine out of 16 light curves over a time span of almost three years, and

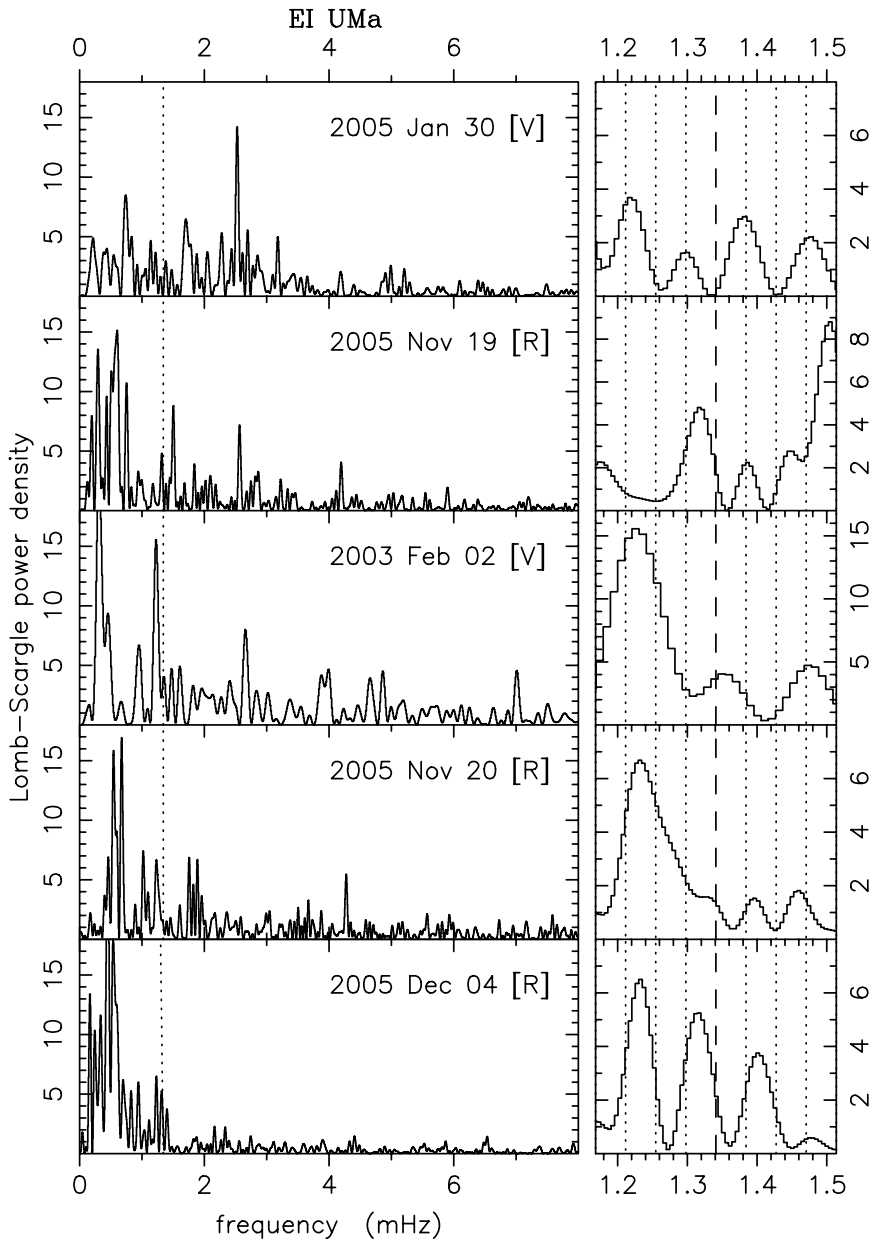

FIG. 4.-Power spectra of prewhitened $V$ - and $R$-band light curves. The two top panels show examples of nondetections, while the three bottom panels show possible detections of a third-order sideband, particularly on 2003 February 2. Compare with Fig. 3.

there is no evidence that the periods change. This suggests that the periodicities are stable, but that the signal amplitude is variable and sometimes drops below our detection threshold. The relative amplitudes of detected periodicities also vary, ranging from $0.4 \%$ to $1.3 \%$. The highest relative $V$-band amplitude was $1.3 \%$ while the lowest $V$-band nondetection limit was $0.6 \%$; therefore, there is at least a factor of 2 change in fractional pulse amplitude. Although we have only a few detections, there is a tendency for the relative pulse amplitude to decrease with increasing system brightness. Enhanced accretion then does not result in a corresponding increase in the pulse amplitude. This suggests that the pulsations arise in a region physically distinct from the accretion disk-a scenario consistent with the intermediate polar interpretation.

\section{PERIODICITY IN ARCHIVAL XMM-NEWTON DATA}

In their spectral analysis of EI UMa using XMM-Newton data, Pandel et al. (2005) comment that they did not find any clearly periodic signal in the X-ray or UV data. To further investigate the presence of an X-ray periodicity, we reexamine the archival $X M M-N e w t o n$ data. To enhance detection, we have separately analyzed the soft $(0.3-2 \mathrm{keV})$ and hard (2-12 keV) bandpasses.

The observations were obtained on 2002 May 10 and 2002 November 2. We concentrate on the medium spectral resolution data obtained with the European Photon Imaging Camera (EPIC), which consists of one PN and two MOS CCD arrays. We have 
reprocessed the observations using SAS 7.0.0 and extracted light curves in $16 \mathrm{~s}$ bins. The May observation was affected by soft proton flares, which significantly increase the particle background rate; these time intervals were eliminated. The background was well behaved for the November observation.

Because the observation duration is shorter for the $\mathrm{PN}$ than for the MOS cameras, we primarily used the combined MOS-1+ MOS-2 light curves for period search (although the PN data yield similar results). The results are presented in Figure 5. A periodicity consistent with $745 \mathrm{~s}$ is clearly detected in the low-energy light curve of the November observation, and marginally detected above $2 \mathrm{keV}$. Since the spin modulations in IPs are energy dependent, with higher amplitudes observed at lower energies, this is not surprising. Strong flickering in the May observations produced red noise that partially obscured the periodicity, but the signal remains present. We cannot claim independent detection of the $745 \mathrm{~s}$ signal in the hard bandpasses or even the soft bandpass in the May data, but the periodograms are consistent with the presence of such a signal, and the November soft bandpass clearly shows a detection. Note that because the XMM-Newton light curves are of shorter duration than the optical light curves, the frequency resolution of the X-ray power spectra is considerably worse. The power spectra shown in Figure 5 have been oversampled and the true period resolution is $\sim 50 \mathrm{~s}$. The detections are therefore consistent with both the spin and the sideband periods, although we strongly favor the spin interpretation.

\section{LONG-PERIOD BRIGHTNESS FLUCTUATIONS}

\subsection{Lack of Orbital Modulation}

To refine the orbital period and tighten the constraints on the system inclination (nominally $23^{\circ}$; Thorstensen 1986), we searched for orbital modulation in the 2005 November-December set of $R$-band photometry. Given the $6.434 \mathrm{hr}$ orbital period of the binary, the secondary star should be relatively bright. With EI UMa designated a dwarf nova, the accretion luminosity should also not overwhelm the secondary star light. We used IRAF's phase dispersion minimization algorithm to search for any modulation.

Using the combined set of light curves, no significant signal was seen near $6.434 \mathrm{hr}$, at half or double this period, or anywhere in the range of 1-20 hr. Removing the mean before combining each night's observation produced the same null result, as well as looking at each light curve individually. Six light curves are shown in Figure 6, folded on the $6.434 \mathrm{hr}$ period; while slow flickering is noticeably present (also see Fig. 7), no periodic signal was found. The lack of any ellipsoidal variations demands a low inclination, consistent with the findings of Thorstensen (1986) and the IUE spectral modeling of Urban \& Sion (2006). Furthermore, the lack of any apparent bright spot also suggests a low inclination, assuming the bright spot is azimuthally elongated and radiates preferentially in the orbital plane. An alternate explanation for the lack of detection of the bright spot is that its light is overpowered by a luminous disk. A bright disk would also explain the lack of any secondary star attributes like ellipsoidal variations or spectral lines, a result that is otherwise hard to explain given that the secondary is likely a K star (Thorstensen 1986). A high mass transfer rate through the disk would imply that EI UMa is more akin to a nova-like cataclysmic variable than a dwarf nova. We return to this point in $\S 6$.

\subsection{Flare Event and Dwarf Nova Status}

Figure 7 shows the seven nights of $R$-band observations taken between 2005 November 18 and December 5. The general shape of the light curve is that of a broad, 0.65 mag amplitude "slow

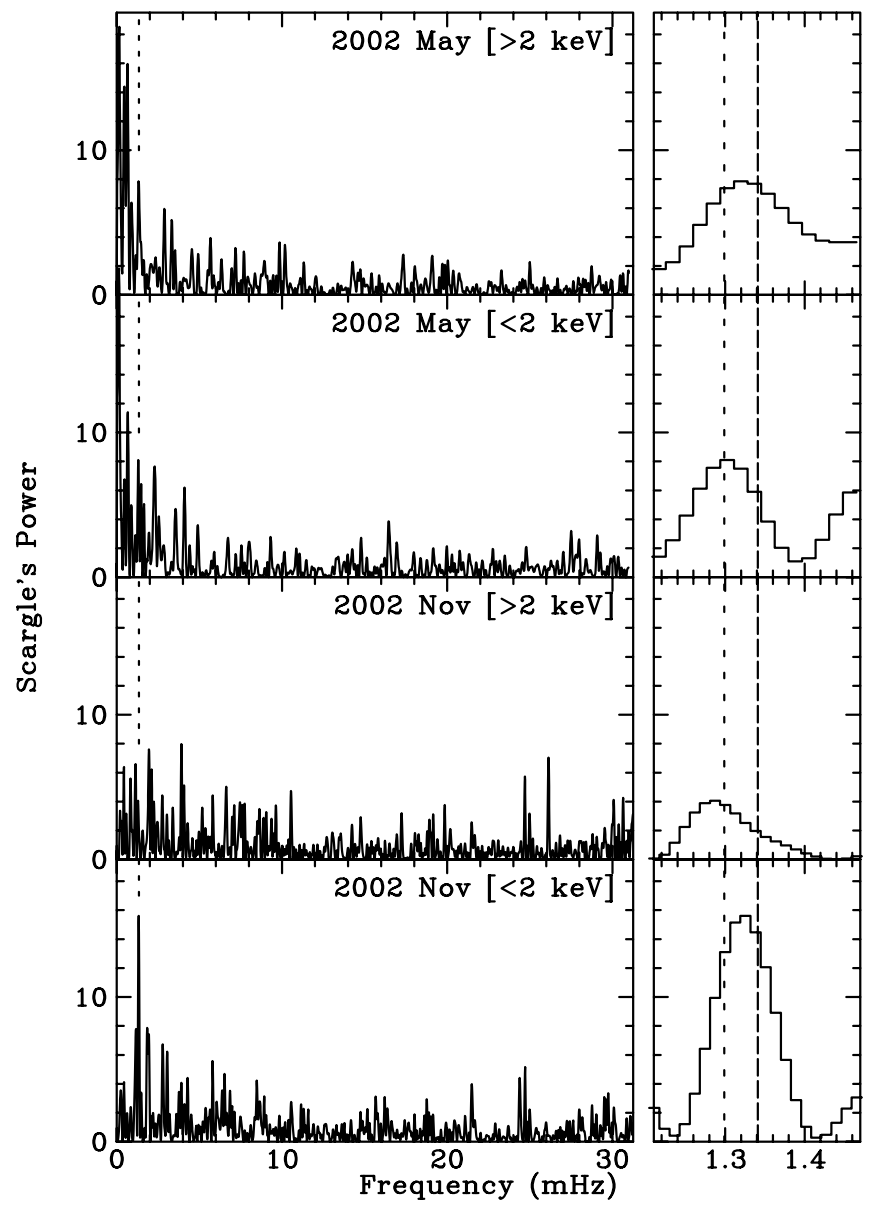

FIG. 5.- Power spectra of XMM-Newton X-ray light curves taken at two epochs and separated into hard and soft bandpasses. The right-hand panels show the region between 680 and $825 \mathrm{~s}$ (equal to the \pm 3 spin-orbit sideband). The dashed line marks the spin period and the dotted line marks the first spin-orbit sideband.

hump" spanning more than 20 days. If we define the $R$-band quiescence level to be $14.55 \mathrm{mag}$ as given by Misselt (1996) the system flux is slightly elevated on November 18 and returns to the quiescent level by December 5. On November 20, on top of this slow variation, is a sharp "fast flare" lasting several hours. In nearly $90 \mathrm{hr}$ of photometry we see no other feature that resembles the fast flare event. The amplitude of the fast flare cannot be determined because the peak was not observed, but a lower limit is $0.27 \mathrm{mag}$, and the average rate of decay was $0.09 \mathrm{mag} \mathrm{hr}^{-1}$ over the observed $3 \mathrm{hr}$ decline. The brightness difference between the minimum on December 5 and the peak of the flare on November 20 was $>0.80$ mag (a factor of $>2.1$ change in flux).

We briefly entertain the hypothesis that the slow hump is a very low amplitude dwarf nova outburst. Warner (1995) gives an empirical outburst decay time as a function of orbital period (his eq. [3.5]):

$$
\tau_{\text {decay }}=0.53 P_{\text {orb }}^{0.84}(h) \text { days } \mathrm{mag}^{-1} .
$$

Using the $6.434 \mathrm{hr}$ orbital period gives a decay timescale of 2.5 days $\mathrm{mag}^{-1}$, and for a small $0.65 \mathrm{mag}$ amplitude outburst, the duration is expected to be only $\sim 1.6$ days - clearly inconsistent with the observed $>10$ day decay of the slow hump. If we include the peak brightness of the flare observed on November 20 , a 0.80 mag amplitude drop in brightness would still usually take only $\sim 2$ days. 


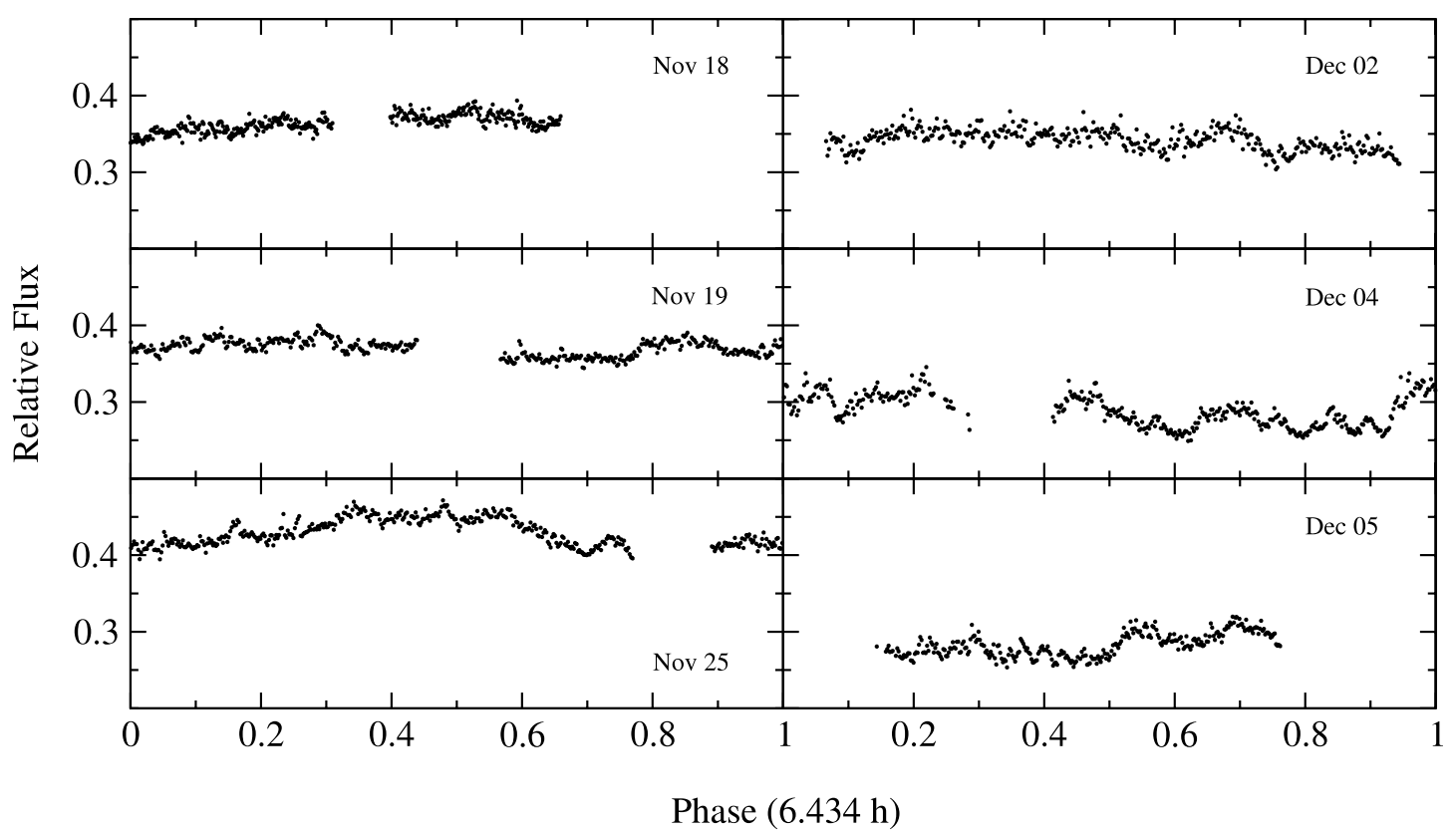

FIG. 6. $-R$-band light curves folded on the $6.434 \mathrm{hr}$ orbital period. The flux is relative to comparison star M3 (Misselt 1996). No obvious periodic modulation on the orbital period is present. In particular, there is no evidence of any bright-spot emission or ellipsoidal variation, suggesting the system has a low orbital inclination, a highmass transfer rate, or both.

Is EI UMa a dwarf nova? AAVSO and VSNet light curves over the last 13 years show substantial day to day variability of $0.5 \mathrm{mag}$ or more, and a slower variation of approximately $1 \mathrm{mag}$ over a timescale of $5 \mathrm{yr}$, but do not reveal any obvious dwarf nova outbursts (see Fig. 1). We searched SIMBAD's list of relevant literature for observations of the mean outburst amplitude, duration, recurrence timescale, etc., but failed to find such information. The evidence suggests that EI UMa's classification as a dwarf nova is suspect.

Some IP systems have exhibited dwarf nova outbursts (e.g., EX Hya, GK Per). Of particular note are three systems that have exhibited short $(<2$ days), low-amplitude $(\$ 2 \mathrm{mag})$ outbursts: V1223 Sgr (van Amerongen and van Paradijs 1989), TV Col (Hellier \& Buckley 1993), and V1062 Tau (Lipkin et al. 2004). It has been suggested that these short outbursts may not be normal disk instability dwarf nova outbursts, but instead are enhanced mass transfer bursts from the secondary star (see Hellier \& Buckley 1993; Hellier et al. 1997; Lipkin et al. 2004). The

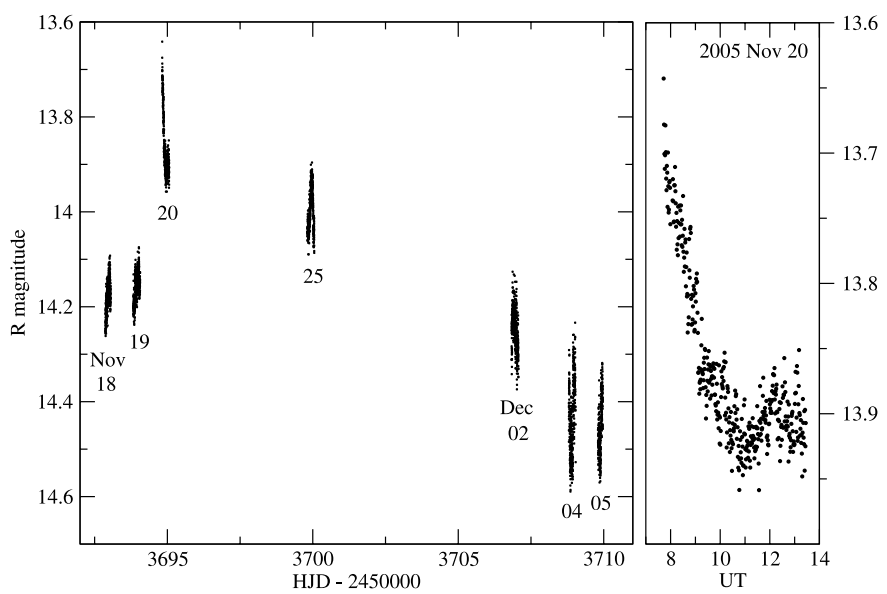

Fig. 7. $-R$-band light curve of the 2005 November/December outburst. Although unresolved, the outburst lasted $\sim 20$ days. Inset: A magnified view of the 2005 November 20 light curve showing the rapid decay of a flare. outburst timescales for TV Col (Hellier \& Buckley 1993) are especially similar to what we have observed with EI UMa. TV Col showed a rapid $\sim 2$ mag flare over several hours in a blue bandpass, followed by a much slower, low-amplitude decay of $\sim 3-5$ days before the system returned fully to preoutburst magnitude levels. The flare exhibited a two stage decay: a sharp decline of $0.6 \mathrm{mag}$ in 40 minutes, followed by slower decline of $0.6 \mathrm{mag}$ over $5 \mathrm{hr}$. The rate of EI UMa's fast flare decay $\left(\sim 0.09 \mathrm{mag} \mathrm{hr}^{-1}\right)$ is comparable with the rate for the latter part of TV Col's decay $\left(\sim 0.12 \mathrm{mag} \mathrm{hr}^{-1}\right)$. By analogy with TV Col, we suggest that the 2005 November-December event may have been a mass transfer burst from the secondary star. Spectroscopic observations of the bright spot and stream emission during a flare could test this hypothesis. In Table 3 we list the outburst properties of the IP systems known to exhibit outbursts, and add EI UMa to the list for comparison.

TABLE 3

Outburst Properties for Intermediate Polar Dwarf Nova Candidates

\begin{tabular}{|c|c|c|c|c|}
\hline System & $\begin{array}{r}P_{\text {orb }} \\
(\mathrm{hr})\end{array}$ & $\begin{array}{c}\text { Duration } \\
\text { (day) }\end{array}$ & $\Delta m$ & Recurrance Interval \\
\hline HT Cam ……........................ & 1.43 & $\sim 2$ & $\sim 4.5$ & $\sim 150$ days? \\
\hline 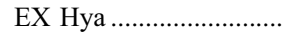 & 1.64 & $2-3$ & $\sim 3.5$ & years \\
\hline 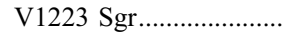 & 3.37 & $\sim 0.5$ & $>1$ & $?$ \\
\hline YY (DO) Dra .................. & 3.96 & $\sim 5$ & $\sim 5$ & $\sim 1000$ days \\
\hline 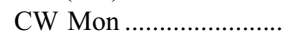 & 4.23 & $\sim 20$ & $\sim 2$ & $100-200$ days \\
\hline 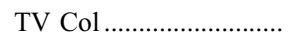 & 5.49 & $\sim 0.5$ & $\sim 2$ & $\sim 1$ month? \\
\hline 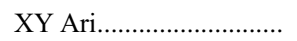 & 6.06 & $\sim 5$ & $\sim 3$ & $\gtrsim 50$ days \\
\hline 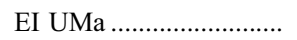 & 6.43 & $\lesssim 1$ & $>0.8$ & $?$ \\
\hline V1062 Tau ......................... & 9.95 & $\sim 1-2$ & $\sim 1.2$ & $\lesssim 6$ months \\
\hline GK Per & 47.9 & $\sim 30-70$ & $\sim 3$ & $880-1240$ days \\
\hline
\end{tabular}

References.-Hellier et al. (1997). TV Col: Hellier \& Buckley (1993). V1223 Sgr: van Amerongen \& van Paradijs (1989). V1062 Tau: Lipkin et al. (2004). HT Cam: Ishioka et al. (2002). EX Hya: Hellier et al. (2000). YY Dra: Wenzel (1983). XY Ari: Hellier et al. (1997). CW Mon: Kato et al. (2003). GK Per: Šimon (2002). 


\section{EI UMa AS AN INTERMEDIATE POLAR}

Having made the case that EI UMa is an IP, we now examine the properties of EI UMa and how it compares with other systems in its class. Of the 26 or so ${ }^{1}$ IPs, EI UMa's 12.4 minute spin period is quite ordinary, being close to the middle of the distribution of spin periods (0.5-120 minutes). EI UMa's $6.43 \mathrm{hr}$ orbital period is longer than about $80 \%$ of cataclysmic variables, and is more typical of higher accretion rate systems than of dwarf novae (e.g., see Shafter 1992). The spin-to-orbital period ratio $P_{\text {spin }} / P_{\text {orb }}$ is 0.03 , smaller than the equilibrium period ratio of $\sim 0.1$ for typical IPs or 0.07 for EI UMa itself(King \& Lasota 1991). Thus it is clear that EI UMa is not in spin equilibrium.

The mass of the secondary star can be estimated from the semiempirical donor star sequence work by Knigge (2006). Extrapolating to just beyond Knigge's donor star sequence table, an orbital period of $6.43 \mathrm{hr}$ has $M_{2}=0.81 \pm 0.05 M_{\odot}, R_{2}=0.76 \pm$ $0.03 R_{\odot}$, and a spectral type of approximately $\mathrm{K} 4$; the errors here are reasonable estimates. It is required that the mass ratio $q \equiv$ $\left(M_{2} / M_{1}\right) \lesssim 1$ for dynamically stable mass transfer, so this places a lower limit on the mass of the white dwarf: $M_{1} \gtrsim 0.81 \pm 0.05 M_{\odot}$. Combined with the white dwarf mass-radius relation (Nauenberg 1972), the upper limit on the white dwarf radius would be $R_{1}=7000 \mathrm{~km}$. A firm upper limit for the mass of the white dwarf is the Chandrasekhar mass limit, but a more reasonable upper limit of $M_{1} \lesssim 1.2 M_{\odot}$ will at times be used for illustrative purposes in the following discussion.

\subsection{Distance}

Thorstensen (1986) states that EI UMa's spectrum showed no absorption features except for "a very marginal feature at $\mathrm{Na} \mathrm{D} \mathrm{...} \mathrm{suggesting} \mathrm{that} \mathrm{less} \mathrm{than} 40 \%$ of the light is from the companion." Using this $40 \%$ limit and a mean magnitude of $V=$ $14.5 \mathrm{mag}$, the secondary star has an apparent magnitude $V_{2}>$ 15.5 mag. The Galactic neutral hydrogen column density ${ }^{2}$ in the direction of EI UMa is $n_{\mathrm{H}}=3.35 \times 10^{20} \mathrm{~cm}^{-2}$, which gives an extinction of $A_{V}<0.187 \pm 0.003$ mag using the ROSAT-derived relation between soft X-ray absorption and optical extinction, as given by Cox (2000). This extinction is highly uncertain, but its inclusion or omission does not substantially affect the discussion that follows.

The absolute magnitude of a K4 V star is $\sim 7.2$ (Gray 2005), so we can estimate a crude minimum distance of $>420 \mathrm{pc}$. A much better distance estimate can be made by using the 2MASS measured $K=13.534$ mag (Skrutskie et al. 2006) and the revised $K$-band Barnes-Evans surface brightness relation as given by Beuermann (2006). This yields an upper limit distance of $<810 \mathrm{pc}$, assuming zero extinction in the $K$ band and that all of the $K$-band light and $40 \%$ of the $V$-band light comes from the secondary star. Beuermann also gives the $K$-band surface brightness in terms of spectral type, which is independent of the observed $(V-K)$ color. Using a K4 spectral type yields a distance of 745 pc. Agreement between the $K$ band and spectral type derived distances can be made if the contribution of the secondary star is $25 \%$. A distance of $\sim 670 \mathrm{pc}$ is derived if the $V$-band surface brightness is used instead, but the $V$ band is much more heavily contaminated by sources of light other than the secondary star, thus making the estimate less reliable. Finally, using Knigge's semiempirical $K$-band absolute magnitude $\left(M_{K} \approx 4.2\right)$ based on the orbital period, a distance of $740 \mathrm{pc}$ is obtained, in good agreement with the above

\footnotetext{
1 For example, see http://asd.gsfc.nasa.gov/Koji.Mukai/iphome/iphome.html. 2 From NASA's HEASARC nH Tool at http:// heasarc.gsfc.nasa.gov/cgi-bin/ Tools/w3nh/w3nh.pl (Dickey \& Lockman 1990).
}

values. Despite the good agreement, we will treat these distance estimates with appropriate caution and adopt a distance of $750_{-200}^{+100} \mathrm{pc}$ in the discussion that follows; note that these error bars represent upper and lower limits, rather than $1 \sigma$ uncertainties.

\subsection{Mass Transfer Rate}

Baskill et al. (2005) measured a $0.8-10 \mathrm{keV}$ X-ray flux of $30.0 \times 10^{-12} \mathrm{ergs} \mathrm{s}^{-1} \mathrm{~cm}^{-2}$; when combined with the above distance, this gives a system $\mathrm{X}$-ray luminosity of $L_{\mathrm{X}} \approx 1.0_{-0.5}^{+0.3} \times$ $10^{33} \mathrm{ergs} \mathrm{s}^{-1}$. Using the conversion from $L_{\mathrm{X}}$ to mass accretion rate given in Warner (1996) $\left(L_{\mathrm{X}}=2.8 \times 10^{15} \dot{M} \mathrm{erg} \mathrm{s}^{-1}\right)$ yields $\dot{M}=3.6_{-1.7}^{+1.0} \times 10^{17} \mathrm{~g} \mathrm{~s}^{-1}$. A second method of finding $\dot{M}$ can be made if we assume the secondary star contributes $\sim 25 \%$ of the $V$-band light, and thus the absolute magnitude of the disk is $M_{V \text { disk }}=5.4_{-0.2}^{+0.7}$. Using the $M_{V}-\dot{M}$ relations in Figure 2 of Smak (1989), the mass accretion rate is $\gtrsim 4.0 \times 10^{17} \mathrm{~g} \mathrm{~s}^{-1}$, in good agreement with the mass accretion rate based on the X-ray luminosity. This range of mass accretion rates is far greater than the boundary layer $\dot{M}$ estimated by Pandel et al. (2005) due to the much smaller distance of $100 \mathrm{pc}$ assumed in that study.

The disk instability model has been relatively successful at reproducing observations of dwarf nova outbursts (e.g., see Warner 1995). The model predicts a critical mass accretion rate, $\dot{M}_{\text {crit }}$, above which the disk remains in a hot, high-viscosity, stable state and the cycle of dwarf nova outbursts is suppressed. This limit has been observationally confirmed (Warner 1995, specifically Fig. 3.9). The expression for the $\dot{M}_{\text {crit }}$ is given by Warner 1996 , eq. [37]:

$\dot{M}_{\text {crit }}=8.26 \times 10^{15}\left(\frac{f}{0.3}\right)^{21 / 8}\left(\frac{\alpha_{\text {hot }}}{0.3}\right)^{3 / 10}(1+q)^{7 / 8} P_{\text {orb }}^{7 / 4} \mathrm{~g} \mathrm{~s}^{-1}$,

where $\alpha_{\text {hot }}$ is the usual viscosity parameterization and $f \equiv r_{\text {disk }} / a$ is the ratio of the outer disk to the orbital separation. The value of $f$ in intermediate polars is probably in the range $0.2-0.3$ (Warner 1996), somewhat smaller than in the nonmagnetic systems; this is because magnetic truncation of the inner disk means less angular momentum needs to be transferred to the outer disk, and hence the outer disk will not grow as large (Angelini \& Verbunt 1989). For EI UMa, $\dot{M}_{\text {crit }}=1-4 \times 10^{17} \mathrm{~g} \mathrm{~s}^{-1}$. If we use the $\dot{M}_{\text {crit }}(P)$ expression from Shafter (1992) we get a similar estimate of $\dot{M}_{\text {crit }} \sim(1-2) \times 10^{17} \mathrm{~g} \mathrm{~s}^{-1}$. Thus, the mass accretion rate we estimate $\left(3.6 \times 10^{17} \mathrm{~g} \mathrm{~s}^{-1}\right)$ is close to, and most likely larger than, the critical mass accretion rate. Such a high $\dot{M}$ is expected to produce a highly luminous disk, explaining why we do not see ellipsoidal variations or a bright spot. The high $\dot{M}$ also explains why dwarf nova outbursts have not been observed: they simply do not occur, and EI UMa is not a dwarf nova.

\subsection{Magnetic Moment}

In order for a cataclysmic variable to be an IP, the white dwarf magnetic moment $\mu$ must be strong enough to disrupt the accretion disk and channel the accretion flow, but not so strong as to completely prevent formation of a disk. Warner (1996) gives this condition as

$$
\frac{\mu_{33}}{\dot{M}_{16}^{1 / 2}}<7.04 \times 10^{-2} \frac{(1+q)^{7 / 12}}{q^{3 / 4}} M_{1}^{5 / 6} P_{\text {orb }}^{7 / 12},
$$

where $\mu_{33}=\mu / 10^{33} \mathrm{G} \mathrm{cm}^{3}$ and $\dot{M}_{16}=\dot{M} / 10^{16} \mathrm{~g} \mathrm{~s}^{-1}$. In addition, the interaction between the white dwarf and secondary star magnetic fields produces a spin-synchronizing torque of strength 
$\zeta$. To be an IP, the magnetic moment must not be so strong as to force synchronous rotation, and this leads to a second condition given by Warner (1996):

$$
\frac{\mu_{33}}{\dot{M}_{16}^{6 / 5}}<0.128\left(\zeta_{\max }\right)^{7 / 5}\left(M_{1}^{2}\right)(1+q)^{7 / 5}\left(P_{\text {orb }}^{-7 / 20}\right),
$$

where $\zeta_{\max }=1.25$. Figure 8 shows the disk and synchronization conditions for the $P_{\text {orb }}$ and $M_{1}$ range of EI UMa.

Warner (1996) notes that IPs observationally tend to cluster near the $\zeta=\zeta_{\max }$ line, as shown in Figure 7 of that work. Assuming this is also true for EI UMa, the range of mass accretion rates given by the X-ray luminosity yields $\mu>(3.4 \pm 0.2) \times$ $10^{33} \mathrm{G} \mathrm{cm}^{3}$ and $B_{1}>9.9 \pm 0.6 \mathrm{MG}$. Typical white dwarf field strengths among IPs are 1-10 MG (Hellier 2001), which puts EI UMa's magnetic field strength at the very high end. Thus, either EI UMa's magnetic field strength is very high for an IP, or the system is an exception to the $\zeta \simeq \zeta_{\max }$ trend. Interestingly, Figure 7 of Warner (1996) shows two other IPs with similarly high magnetic moments and mass accretion rates: V1223 Sgr and V1062 Tau, two of the three other systems also thought to exhibit mass transfer bursts from the secondary, as mentioned in $\S 5$.2. Using the illustrative upper limit for the white dwarf mass yields a magnetic field strength of $170 \mathrm{MG}$, well above the typical range for IPs. A magnetic field of this strength is problematic, since spectra taken by Thorstensen (1986) do not show any hint of cyclotron harmonics or Zeeman splitting expected with such high $B_{1}$ values. Since higher $B_{1}$ values correlate with smaller radii and higher $M_{1}$ values, the white dwarf mass is probably be closer to the lower mass limit.

A strong magnetic field moves the inner radius of the disk,

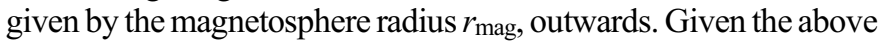
parameters and equation (6.5) of Warner (1995) we find $r_{\text {mag }}>$ $(1.2-1.3) \times 10^{10} \mathrm{~cm}$. There is still room for a disk to form however, since the circularization radius $r_{\text {circ }}>(1.7-1.8) \times 10^{10} \mathrm{~cm}$ (Frank et al. 2002, eq. [4.20]) is greater than $r_{\text {mag. The fastness }}$ parameter $P_{\mathrm{Kep}} / P_{\text {spin }} \gtrsim 1.1$, where $P_{\mathrm{Kep}}$ is the Keplerian period at $r_{\text {mag }}$. Because $P_{\text {Kep }} / P_{\text {spin }}>1$, there is likely some propeller component to EI UMa's accretion. Since the $\zeta \approx \zeta_{\max }$ assumption brings EI UMa close to the disk/no disk boundary of Figure 8, accretion in the EI UMa system may look less like a disk and more like an "accretion ring." Given the long orbital period, high magnetic moment, and ring-shaped accretion disk, we speculate that EI UMa will eventually evolve into a diskless system as the orbital separation slowly decreases. Hence, EI UMa appears to be the precursor to a polar.

\subsection{Hidden Intermediate Polars}

Prior to this work, several researchers suggested that EI UMa is an IP (e.g., Baskill et al. 2005; Pandel et al. 2005; Thorstensen 1986). Our discovery of optical pulsations at the spin and spinorbit sideband have confirmed these suspicions. This has led us to wonder if the heavy reliance on the detection of a stable pulsation for defining an IP may be too conservative, and that we are failing to recognize members of the IP class. If true, this would affect statistical studies of the IP population as a whole. In the worst case scenario, we may be recognizing and studying only the most spectacular members of the class, leading to a bias in our understanding of the X-ray and optical spin modulation mechanisms.

Baskill \& Wheatley (2006) propose that LS Peg is such a "hidden IP"; its X-ray spectral characteristics are IP-like, yet it shows no permanent pulsation. This is in spite of its periodic circular polarization and $\mathrm{H} \beta$ flaring, indicating it is an IP

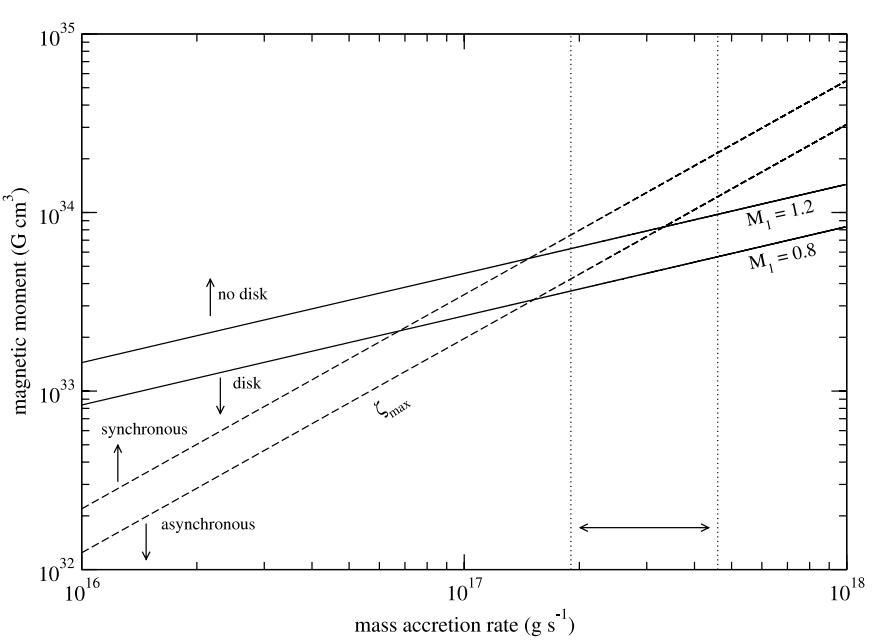

FIG. 8.-Magnetic moment vs. mass accretion rate plot for $P_{\text {orb }}=6.434 \mathrm{~h}$, and for $M=0.8 M_{\odot}$ or $1.2 M_{\odot}$. The disk condition is marked by solid lines, while the synchronization condition is marked by dashed lines. The $\dot{M}$ range for EI UMa is bounded by the vertical dotted lines.

(Rodríguez-Gil et al. 2001). The authors point out that the degree of misalignment between the spin and magnetic axes of the white dwarf is an important factor in the production of a modulation: if the two axes are nearly aligned, any photometric modulations on the spin period would become much weaker, perhaps undetectable. V426 Oph is another CV with a highly absorbed $\mathrm{X}$-ray spectrum, a characteristic of IPs, and hence is thought to be an IP (Baskill et al. 2005). We note that TW Pic (Norton et al. 2000) may be considered a third candidate hidden IP. EI UMa sometimes exhibits a pulse amplitude too low to detect, and could also be called a hidden IP. The existence of four such systems suggests a sizable population of hidden IPs may exist, perhaps as many as $15 \%(=4 / 26)$ of all IPs. The above simple argument hints that the spin and magnetic field axes may be aligned in roughly $15 \%$ of cataclysmic variable white dwarfs.

\section{CONCLUSIONS}

Our main conclusions are the following:

1. We have detected stable, $\sim 1 \%$ amplitude oscillations in optical photometry near the X-ray oscillation period discovered by Baskill et al. (2005). We attribute a $U$-band periodicity near $745 \mathrm{~s}$ to the white dwarf spin, and a $V$ - and $R$-band periodicity near $770 \mathrm{~s}$ to the spin-orbit sideband. A periodicity at $\sim 813 \mathrm{~s}$ is sometimes present in the $V$ and $R$ bands and may be related to the third spin-orbit sideband (expected at $821 \pm 3$ s), but the association is tentative. The relative signal amplitude is variable by at least a factor of 2 .

We have also detected a periodicity in archival XMM-Newton observations consistent with the 745 s periodicity. The $X M M$ Newton observations suggest the X-ray oscillation is stronger at softer energies $(<2 \mathrm{keV})$ than at hard energies. The $U$-band periodicity agrees with both X-ray periodicity detections, and combined give a spin period of $745.7 \pm 2.7 \mathrm{~s}$. Combined with other evidence from previous X-ray observations (Pandel et al. 2005; Baskill et al. 2005), our observations strongly suggest that EI UMa is an intermediate polar.

2. An outburst event was observed in 2005 NovemberDecember with an amplitude $>0.65$ mag in the $R$ band and a full duration of at least 20 days. The decline of a flare on top of the longer timescale outburst event was observed on 2005 November 20 , in which the $R$-band brightness dropped by 0.27 mag in $3 \mathrm{hr}$. The low amplitude and long duration of the outburst are 
uncharacteristic of normal dwarf novae. We argue that it is unlikely that EI UMa is a dwarf nova, a conclusion supported by AAVSO and VSNet observations going back to 1993 and by the high mass transfer rate we derive. By analogy with TV Col, we interpret the outburst and the flare as mass transfer events from the secondary star.

3. Using the Barnes-Evans surface brightness relation, we estimate the distance to EI UMa is $750_{-200}^{+100} \mathrm{pc}$. The disk mass transfer rate $\dot{M} \simeq 3.6 \times 10^{17} \mathrm{~g} \mathrm{~s}^{-1}$, likely greater than the critical rate above which dwarf nova outbursts are prevented. Assuming EI UMa exhibits typical IP behavior, the magnetic moment is $(3.4 \pm 0.2) \times 10^{33} \mathrm{G} \mathrm{cm}^{3}$. This strong magnetic moment, paired with a long orbital period, implies that EI UMa contains an accretion ring rather than a disk. We speculate that EI UMa is a prepolar and will evolve into a diskless cataclysmic variable.
We thank Nassissie Fedaku and Danny Martino for assisting with the observations in 2003, and Allen Shafter and the referee for valuable comments. We also gratefully acknowledge the variable star observers with the AAVSO and the VSNet, whose longterm observations were used in this research. This research has made use of NASA's Astrophysics Data System and the SIMBAD database, operated at CDS, Strasbourg, France. IRAF is distributed by the National Optical Astronomy Observatories, which are operated by the Association of Universities for Research in Astronomy, Inc., under cooperative agreement with the National Science Foundation. Support for this research was provided by NASA through grants HST-GO-09078.01A and HST-GO08156.02A from the Space Telescope Science Institute, which is operated by the Association of Universities for Research in Astronomy, Incorporated, under NASA contract NAS5-26555.
Angelini, L., Verbunt, F. 1989, MNRAS, 238, 697

Baskill, D. S., \& Wheatley, P. J. 2006, in Proc. X-Ray Universe 2005, ed. A. Wilson (ESA SP-604; Noordwijk: ESA), 239

Baskill, D. S., Wheatley, P. J., \& Osborne, J. P. 2005, MNRAS, 357, 626

Beuermann, K. 2006, A\&A, 460, 783

Cook, M. C. 1985, MNRAS, 215, 81

Cox, A. N. 2000, Allen's Astrophysical Quantities (4th ed.; New York: Springer

Dickey, J. M., \& Lockman, F. J. 1990, ARA\&A, 28, 215

Frank, J., King, A., \& Raine, D. 2002, Accretion Power in Astrophysics (3rd ed.; Cambridge: Cambridge Univ. Press)

Gray, D. F. 2005, The Observation and Analysis of Stellar Photospheres (3rd ed.; Cambridge: Cambridge Univ. Press)

Green, R. F., Ferguson, D. H., Liebert, J., \& Schmidt, M. 1982, PASP, 94, 560

Hassall, B. J. M., et al. 1981, MNRAS, 197, 275

Haswell, C. A., Patterson, J., Thorstensen, J. R., Hellier, C., \& Skillman, D. R. 1997, ApJ, 476, 847

Hellier, C. 2001, Cataclysmic Variable Stars: How and Why They Vary (Chichester: Springer Praxis)

Hellier, C., \& Buckley, D. A. H. 1993, MNRAS, 265, 766

Hellier, C., Kemp, J., Naylor, T., Bateson, F. M., Jones, A., Overbeek, D., Stubbings, R., \& Mukai, K. 2000, MNRAS, 313, 703

Hellier, C., Mukai, K., \& Beardmore, A. P. 1997, MNRAS, 292, 397

Henden, A. A., \& Honeycutt, R. K. 1997, PASP, 109, 441

Ishioka, R., et al. 2002, PASJ, 54, 581

Kato, T., et al. 2003, PASJ, 55, 491

King, A. R., Lasota, J.-P. 1991, ApJ, 378, 674

Knigge, C. 2006, MNRAS, 373, 484

Lipkin, Y. M., Leibowitz, E. M., \& Orio, M. 2004, MNRAS, 349, 1323

\section{REFERENCES}

Misselt, K. A. 1996, PASP, 108, 146

Nauenberg, M. 1972, ApJ, 175, 417

Norton, A. J., Beardmore, A. P., Retter, A., \& Buckley, D. A. H. 2000, MNRAS, 312, 362

Osborne, J. P., Rosen, R. Mason, K. O., \& Beurmann, K. 1985, Space Sci. Rev., 40, 143

Pandel, D., Córdova, F. A., Mason, K. O., \& Priedhorsky, W. C. 2005, ApJ, 626,396

Patterson, J. 1994, PASP, 106, 209

Patterson, J., \& Price, C. M. 1981, ApJ, 243, 83

Patterson, J., Schwartz, D. A., Pye, J. P., Blair, W. P., Williams, G. A., \& Caillault, J.-P. 1992, ApJ, 392, 233

Press, W. H., Teukolsky, S. A., Vetterling, W. T., \& Flannery, B. P. 2003, Numerical Recipes in Fortran (2nd ed.; Cambridge: Cambridge Univ. Press)

Ritter, H., \& Kolb, U. 2003, A\&A, 404, 301

Rodríguez-Gil et al. 2001, ApJ, 548, L49

Shafter, A. W. 1992, ApJ, 394, 268

Šimon, V. 2002, A\&A, 382, 910

Skrutskie, M. F., et al. 2006, AJ, 131, 1163

Smak, J. 1989, Acta Astron., 39, 317

Thorstensen, J. R. 1986, AJ, 91, 940

Urban, J. A., \& Sion, E. M. 2006, ApJ, 642, 1029

van Amerongen, S., \& van Paradijs, J. 1989, A\&A, 219, 195

Warner, B. 1989, Inf. Bull. Variable Stars, 3383, 1

1995, Cataclysmic Variable Stars (Cambridge: Cambridge Univ. Press) 1996, Ap\&SS, 241, 263

Wenzel, W. 1983, Inf. Bull. Variable Stars, 2262, 1 